\title{
Raman study on single-walled carbon nanotubes with different laser excitation energies
}

\author{
S S ISLAM*, KHURSHED AHMAD SHAH, H S MAVI ${ }^{\dagger}$, A K SHAUKLA ${ }^{\dagger}$, \\ S RATH ${ }^{\ddagger}$ and HARSH ${ }^{\#}$ \\ Department of Applied Sciences and Humanities, Faculty of Engineering and Technology, Jamia Millia Islamia \\ (Central University), New Delhi 110 025, India \\ ${ }^{\dagger}$ Department of Physics, Indian Institute of Technology, New Delhi 110 016, India \\ ${ }^{\ddagger}$ Department of Physics and Astrophysics, Delhi University, Delhi 110 007, India \\ \#Solid State Physics Laboratory, New Delhi 110 054, India
}

MS received 4 December 2006; revised 4 April 2007

\begin{abstract}
The industrial use of carbon nanotubes is increasing day by day; therefore, it is very important to identify the nature of carbon nanotubes in a bundle. In this study, we have used the Raman spectroscopic analysis on vertically aligned single-walled carbon nanotubes (SWCNTs) grown by the chemical vapour deposition (CVD) technique. The grown sample is excited with two laser excitation wavelengths, $633 \mathrm{~nm}$ from $\mathrm{He}-\mathrm{Ne}$ laser and $514.5 \mathrm{~nm}$ from $\mathrm{Ar}^{+}$laser. Raman spectrum in the backscattering geometry provides the characteristic spectra of SWCNTs with its radial breathing mode (RBM), defect-induced disorder mode (D band), and highenergy modes ( $G$ and $M$ bands). The Raman signal positions of the spectra in RBM, $G$ and $M$ bands confirm the grown sample to be of semiconducting type in nature.
\end{abstract}

Keywords. SWCNTs; wavelength dependent spectra; Raman spectroscopy.

\section{Introduction}

The chirality of a single-walled carbon nanotube determines whether the tube is metallic or semiconducting (Dresselhaus et al 1996). Both semiconducting as well as metallic carbon nanotubes (CNTs) find a number of potential applications (Trans et al 1998; Kong et al 2000; Javey et al 2003), however, their potential applications will go high on obtaining either purely metallic or semiconducting carbon nanotubes (Dai 2002). In recent years, efforts have been made to identify the nature of single-walled carbon nanotubes while surveying the electronic properties of carbon nanotubes (Wooning et al 2002; Islam and Shah 2006). For large-scale synthesis of carbon nanotubes, the most promising technique is chemical vapour deposition (CVD) of carbon molecular precursors, assisted by the catalytic activity of metal or transition metal particles (Laurent et al 1998). The CVD technique yields high percentage of semiconducting single-walled carbon nanotubes (S-SWCNT), which is much higher than those obtained using laser ablation (Wooning et al 2002). In this study, growth of vertically aligned single-walled carbon nanotubes is carried out using thermal catalytic CVD technique at $\sim 750^{\circ} \mathrm{C}$ where $\mathrm{CNT}$ is grown on deposited iron catalyst particles using hydrocarbons as precursor.

\footnotetext{
*Author for correspondence (safiul_el@rediffmail.com)
}

Density function theory and first principle calculations are the tools which are used for the identification of the nature of single-walled carbon nanotubes (Laurent et al 1998; Zhou et al 2000; Wooning et al 2002; Islam and Shah 2006). Experimentally Raman spectroscopy is a nondestructive, non-dissipative measurement, which is made at ambient conditions. In particular, resonance Raman spectroscopy can select those nanotubes in the nanotube sample, which are resonant with excitation laser energy, thus, we can observe the properties of a nanotube by resonance Raman spectroscopy even for a bundle of nanotubes. The analysis is carried out in low energy phonon spectra, normally called radial breathing mode (RBM), $\sim 150$ $400 \mathrm{~cm}^{-1}$ and the high energy modes (HEM) at $\sim 1600 \mathrm{~cm}^{-1}$. The study may help in characterization of preferential growth of semiconducting single-walled carbon nanotubes for their potential use in building arrays of field effect transistors and sensors.

\section{Experimental}

Raman scattering spectra are taken from the sample in backscattering geometry; the beam was focused to a circular spot of $1 \mu \mathrm{m}$ diameter. The sample was irradiated with two wavelengths, $633 \mathrm{~nm}$ from $\mathrm{He}-\mathrm{Ne}$ laser and $514.5 \mathrm{~nm}$ from $\mathrm{Ar}^{+}$-laser. The Raman spectra were recorded by employing a spectroscopic system consisting of a 
double monochromator (SPEX 1404), photomultiplier tube (9214B) as detector, an amplifier-discriminator assembly, notch-filter, and a photon counting arrangement. To enhance the signal, an objective lens, $100 \times$, was used. SEM measurements were carried out by scanning electron microscope (LEO-1450 UP).

\section{Results and discussion}

\subsection{Sample growth and its morphology}

Aligned carbon nanotubes (CNTs) were prepared using CVD technique. Growth of carbon nanotubes was carried out using thermally heated chemical vapour deposition reactor having three independent process gas lines for sequential or simultaneous flow of gases. The flow of gases was controlled through mass flow controllers. The temperature profile of the reactor tube was measured using a $\mathrm{K}$ type (Ni-Cr and $\mathrm{Ni}-\mathrm{Al}$ ) thermocouple to fix constant temperature zone inside the reactor. To start with, the substrate was precleaned to remove any greasy materials from the surface. Iron catalyst films of various thicknesses ranging from 10-20 nm were deposited onto a $n$-type Si (100) substrate of resistivity $(\rho) \sim 4-6 \Omega \mathrm{cm}$, using standard RF sputtering technique. The thickness of the film was measured using a surface profiler. The catalyst coated substrate was kept over quartz holder in the CVD reactor and heated at about $650^{\circ} \mathrm{C}$ for $20 \mathrm{~min}$ in $\mathrm{H}_{2}$ flow of $100 \mathrm{sccm}$. After pretreatment of the substrate, $\mathrm{C}_{2} \mathrm{H}_{2}$ was introduced into the reactor and growth of carbon nanotubes was carried out with a $10: 1$ mixture of $\mathrm{H}_{2}$ and $\mathrm{C}_{2} \mathrm{H}_{2}$ for about $8 \mathrm{~min}$ duration. The substrate surface was scanned by using SEM. Figure 1 shows the SEM micrograph where growth of vertically aligned SWNTs is observed.

\subsection{Raman spectroscopic analysis}

The Raman spectra were taken in the backscattering geometry on the sample as grown without having any synthe-

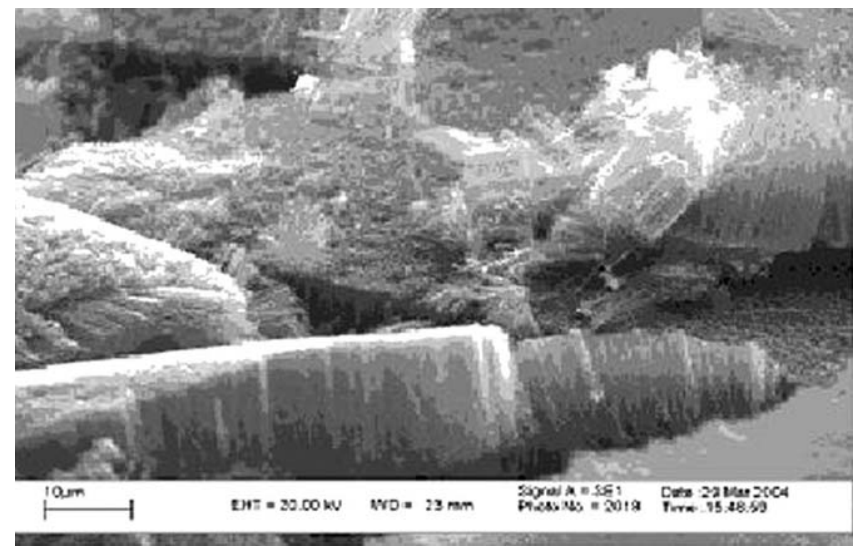

Figure 1. SEM image of single-walled carbon nanotubes (SWCNTs) sis for purification as mentioned. Figure 2 shows the room temperature Raman spectra of SWNT material at a laser excitation energy of $1.96 \mathrm{eV}(\lambda=633 \mathrm{~nm}$ from He-Ne). The spectra is divided into four main zones: the low frequency region from $140-500 \mathrm{~cm}^{-1}$, the intermediate zone from $500-1000 \mathrm{~cm}^{-1}$, the high frequency zone from $1000-2000 \mathrm{~cm}^{-1}$ and second order scattering zone from 2000-3200 $\mathrm{cm}^{-1}$ (figure 2). The first three zones are the characteristics of SWCNTs and distinguish it from other carbon materials either in bulk or nanoform.

Two peculiarities make these spectra different from the spectrum of a planar graphite: (i) presence of RBM modes in the low frequency zone which is earmarked as a silence zone for graphite and other carbonaceous materials, and (ii) the splitting of $\mathrm{G}$ band at $\sim 1600 \mathrm{~cm}^{-1}$ (Rao et al 1997). The phonon modes, $<400 \mathrm{~cm}^{-1}$, are called acoustic modes. Among them the phonons of frequency in the range 150$220 \mathrm{~cm}^{-1}$ are called radial breathing modes (RBM) which are most sensitive (Rao et al 1997) and act as indicator about the quality of SWCNTs and its geometry.

3.2a Radial breathing modes (RBM): Radial breathing mode is conceptually the easiest mode to understand in carbon nanotubes. This mode has $A_{1 \mathrm{~g}}$ symmetry and all the carbon atoms move in phase in the radial direction creating breathing like vibration of the entire tube (Rao et al 1997). The RBM is associated with a periodicity imposed on a graphene sheet by wrapping it into a finite-size (small diameter) tube. It is widely applied to determine the diameter of nanotubes as it has particularly a simple relationship between its frequency and the diameter of the tube (Rao et al 1997; Jorio et al 2003). Also the mode shifts to higher wave numbers when SWCNTs are close packed into bundles, an effect associated with van der Waals forces, acting between nanotubes in a bundle (Rao et al 1997). The RBM ( $\left.\omega_{\mathrm{RBM}}\right)$, the first order Raman modes with phonon wave vector, $q \approx 0$, appears at $162,186,216$, 252 and $433 \mathrm{~cm}^{-1}$. The diameters are calculated using the relationship between $\omega_{\mathrm{RBM}}$ and the tube diameter for SWCNTs (Jorio et al 2003). The diameter range comes out to be $1 \cdot 382-0 \cdot 516 \mathrm{~nm}$.

The nature of a tube in a bundle can be determined if a number of wavelengths are utilized (Rao et al 1997). Figure 3 depicts RBM spectra at room temperature using two laser wavelengths, $633 \mathrm{~nm}$ and $514.5 \mathrm{~nm}$, on the same sample. It is known that SWCNTs can be truly metallic $(n, n)$ armchair SWCNTs with $m-n=3 \times$ integer and semiconducting $(m, n)$ SWCNTs (S-SWCNTs) with $m-n \neq 3 \times$ integer. To confirm that our sample is of semiconducting type, we carried out theoretical calculations at peak positions shown by both wavelengths. Figure 3 shows four peak positions at $180,190,216$ and $252 \mathrm{~cm}^{-1}$. Using the equation (Kurti et al 2002),

$$
\omega_{\mathrm{RBM}}=\left(A / d_{\mathrm{t}}\right)+B
$$




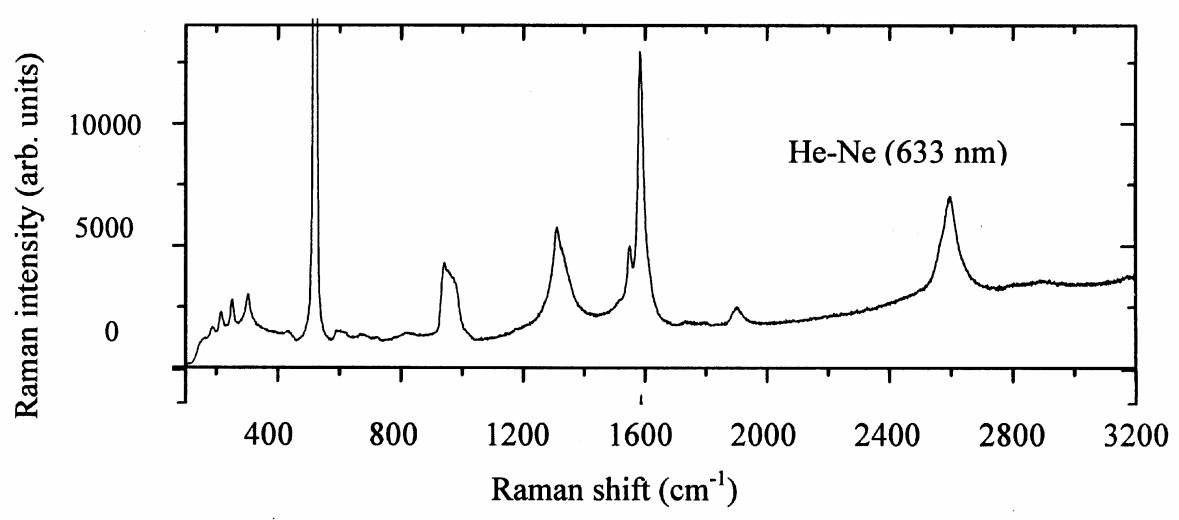

Figure 2. Room temperature Raman spectra of grown SWCNT material at a laser excitation energy, $1.96 \mathrm{eV}(\lambda=633 \mathrm{~nm}$ from He-Ne laser $)$.

where $\omega_{\mathrm{RBM}}$ is RBM frequency, $d_{\mathrm{t}}$ the tube diameter and $A$ and $B$ are constants, and for bundle $A=234 \mathrm{~cm}^{-1}$, $B=10 \mathrm{~cm}^{-1}$, the corresponding tube diameters are 1.376 , $1.3,1.135$ and $0.966 \mathrm{~nm}$, respectively. Within $5 \%$ error range in tube diameters in RBM, as presented earlier by Namkung et al (2004), with no preference in chirality, we find that the sample contains high percentage of semiconducting single-walled carbon nanotubes and this confirms that our sample is of semiconducting type in nature.

$3.2 \mathrm{~b}$ Intermediate zone $\left(500-1000 \mathrm{~cm}^{-1}\right)$ : This region has few peaks at 520, 590,668,726, 824 and $948 \mathrm{~cm}^{-1}$ (figure 2). The peak at $520 \mathrm{~cm}^{-1}$ is due to Si substrate; and the other peaks can be identified as overtones and combinations of lower frequency modes (Journet et al 1997). In the range $750-800 \mathrm{~cm}^{-1}$, Rao et al (1997) identified these peaks with phonon modes scattered from tubes of different diameters with different chiral index. So far very few workers have reported the appearance of intermediate modes in case of SWNTs (Journet et al 1997; Rao et al 1997; Wei et al 2003).

3.2c High energy modes (HEM) $\left(1000-2000 \mathrm{~cm}^{-1}\right)$ : This region contains mainly two prominent modes: the defectinduced disordered mode (D-band) at $1312 \mathrm{~cm}^{-1}$, and tangential modes (G-band) with splitting of three modes at 1516,1552 and $1584 \mathrm{~cm}^{-1}$. Besides, two other modes (M-band) appear at 1732 and $1903 \mathrm{~cm}^{-1}$ (figure 2). The higher energy modes are not strongly dependent on nanotube diameter but they are very sensitive to the local movements of the atoms (Saito et al 1998). We have given here explanation of the characteristics of each band.

D-band $\left(1332 \mathrm{~cm}^{-1}\right)$ : The D-mode at $1350 \mathrm{~cm}^{-1}$ has been known in graphite for over a few decades and this is induced by disorder and not a $\Gamma$-point phonon (Tuinstra and Koenig 1970). In carbon nanotubes, this D-mode is observed at somewhat smaller frequency than in graphite and shifts at a rate between 38 and $65 \mathrm{~cm}^{-1} / \mathrm{eV}$ with laser excitation energy (Kastner et al 1994; Thomson 2000; Kurti et al 2002; Maultzsch et al 2002).

In figure 2 , the D-mode is observed at $1332 \mathrm{~cm}^{-1}$, usually lower than D-mode frequency from graphite and its intensity is quite large as compared to G-band. The bandwidth is $\sim 40 \mathrm{~cm}^{-1}$. These two characteristics (frequency and linewidth) ensure that the sample is nanotube and not graphite (Rao et al 1997). The observation of large Dband peaks compared with the G peak intensity in SWCNTs bundles indicate the presence of amorphous carbon (Rao et al 1997; Ferrari and Robinson 2000). The origin of Dmode is explained in depth in literature (Thomsen and Reich 2000; Maultzsch et al 2001; Saito et al 2003) in terms of double resonance process and it enhances the intensity of the second order bands such as those labeled as $M$ and $M^{+}$that appear in the range $1700-1800 \mathrm{~cm}^{-1}$ and those close to 2700 or $3200 \mathrm{~cm}^{-1}$ (Brar et al 2002) or even higher energies (Tan et al 1998; Cansado et al 2002). The explanation of the shift as a function of laser excitation energy may be due to the selective enhancement of different tubes in resonant Raman scattering (Kastner 1994; Alvarez et al 2000).

G-band $\left(1516-1585 \mathrm{~cm}^{-1}\right)$ : In graphite and carbon nanotubes, the G-band Raman vibrational modes $\left(E_{2 \mathrm{~g}}\right.$ derived modes) are present due to the in-plane vibrational movement of carbon atoms. The observation of characteristic multi-peak features around $1580 \mathrm{~cm}^{-1}$ provides a signature of carbon nanotubes. Unlike graphite (tangential mode at $1582 \mathrm{~cm}^{-1}$ ), the tangential G-mode in SWCNTs gives rise to a multipeak feature, also named the G-band. The two most intense $G$ peaks are labeled $\mathrm{G}^{+}$for atomic displacement along the tube axis, and $\mathrm{G}^{-}$for modes with atomic displacement along the circumferential direction and the lowering of the $\mathrm{G}^{-}$mode is due to the curvature of the nanotube, which softens the tangential vibration in the circumferential direction (Rao et al 1997). Jorio et al 


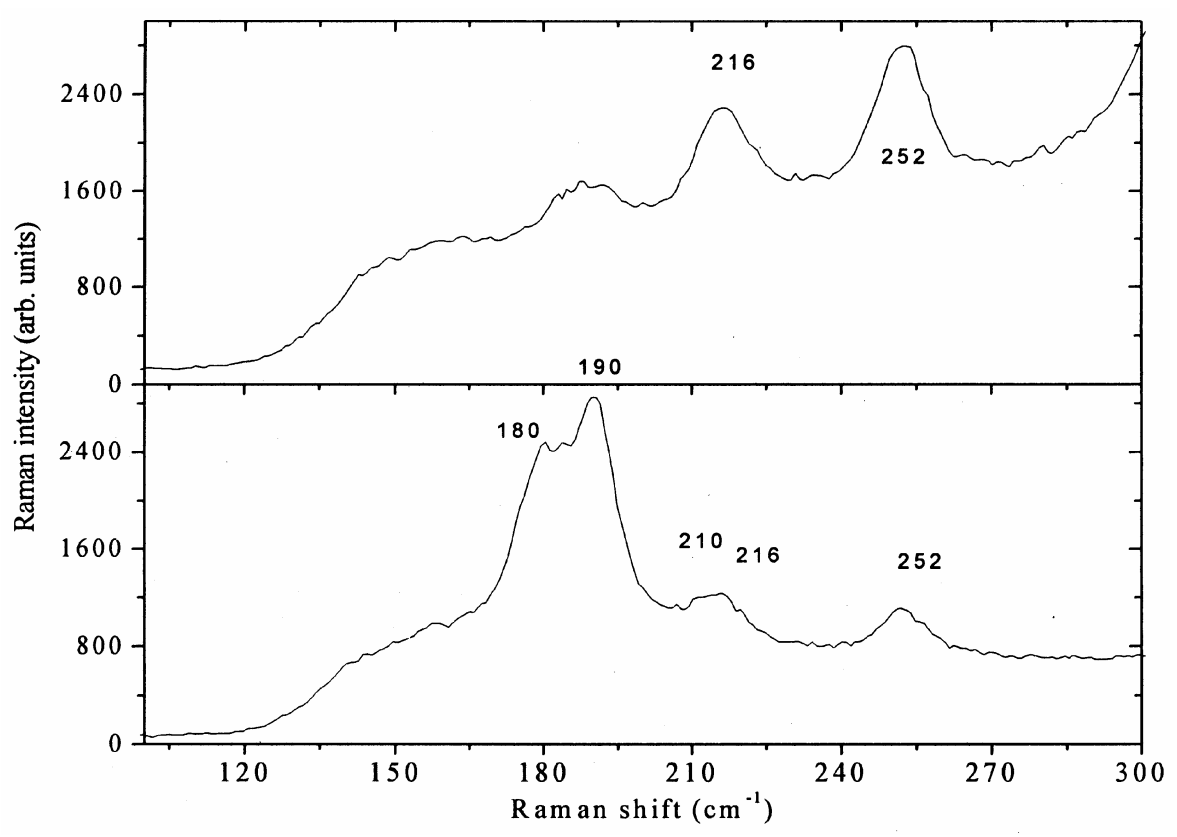

Figure 3. Wavelength dependent radial breathing mode (RBM) spectra of SWCNTs.

(2000) observed the G-band with a prominent mode around $1592\left(\mathrm{G}^{+}\right)$along with two shoulders in the low energy side for SWNT samples.

Our spectra (figure 2) shows $\mathrm{G}$ band with peak position at $1584\left(\mathrm{G}^{+}\right)$of maximum intensity and two shoulders at $1552\left(\mathrm{G}^{-}\right)$and $1516 \mathrm{~cm}^{-1}$. These features have previously been reported in the literature (Rao et al 1997; Jorio et al 2000; Thomsen et al 2000), where the spectral profile resembles our spectra but with a difference in the peak positions. Our spectra (figure 2) resemble the Raman spectra (Tan et al 1998; Thomsen et al 2000; Shirolshi et al 2003) and it is of semiconducting type. They typically arise at laser energies around the transition energies between the singularities in the electron density of states (EDOS) for semiconducting tubes (Rao et al 1997). The Breight-Weigner-Fano (BWF) line is observed in many graphite-like materials with metallic character as well as metallic SWCNTs. The BWF line at $1540 \mathrm{~cm}^{-1}$ which is the characteristic of metallic type, is absent in the spectra. This, in turn, reinforces our suggestion that the grown nanotubes are of semiconducting type and not metallic. In MWNT, there is a single peak at $1589 \mathrm{~cm}^{-1}$ corresponding again to the high-energy graphite like mode; the low energy RBM mode is generally not seen (Thomsen et al 2000). It also confirms that the grown tubes are not even multi-walled carbon nanotubes (MWCNTs).

M-band $\left(1600-2000 \mathrm{~cm}^{-1}\right): \quad$ The spectra (figure 2) shows appearance of two peaks at 1732 and $1900 \mathrm{~cm}^{-1}$ in the high frequency region. The band at $1732 \mathrm{~cm}^{-1}$ is termed as Mband (Brar et al 2002; Sanjuan et al 2004). In our case, the mode frequency of $\mathrm{M}$ band are in agreement with Brar et al (2002) though our laser excitation energy is
$1.96 \mathrm{eV}$. The shift rate is $-3 \mathrm{~cm}^{-1} / \mathrm{eV}$ with increasing laser excitation energy (Brar et al 2002). Therefore, the shift of M-band with laser excitation energy is considered to be negligible in our case. The origin of the mode at $1732 \mathrm{~cm}^{-1}$ is explained as due to the combination of $1516 \mathrm{~cm}^{-1}$ of $\mathrm{G}$-band mode and the radial breathing mode at $216 \mathrm{~cm}^{-1}$. The origin of the mode at $1900 \mathrm{~cm}^{-1}$ is due to the overtone of the mode at $948.9 \mathrm{~cm}^{-1}$ in the intermediate range.

\section{Conclusions}

Vertically aligned single-walled carbon nanotubes (SWCNTs) are grown on Si-substrate using iron catalyst under thermally heated chemical vapour deposition technique (CVD). In order to determine the nature of the nanotubes in the grown bundle sample, the sample was excited by two-laser excitation wavelengths, $633 \mathrm{~nm}$ from $\mathrm{He}-\mathrm{Ne}$ laser and $514.5 \mathrm{~nm}$ from $\mathrm{Ar}^{+}$. Raman spectroscopic analysis at peak positions in radial breathing mode (RBM) and high energy modes (HEMs) confirms that the excited sample has high percentage of semiconducting single-walled nanotubes. Moreover, the study provides an alternative method for the identification of nature of single-walled carbon nanotubes in a bundle for their potential use in consumer electronics.

\section{Acknowledgement}

The authors are highly thankful to Dr P K Basu and his CNT group at the Solid State Physics Laboratory, Delhi, for allowing us to carry out experimental work in their laboratory. 


\section{References}

Alvarez L, Righi A, Rols S, Anglaret E and Sauvajol J L 2000 Chem. Phys. Lett. 320441

Brar V W et al 2002 Phys. Rev. B66 155418

Cansado L G et al 2002 Phys. Rev. B66 155418

Dai H S 2002 Science $\mathbf{5 0 0} 218$

Dresselhaus M S, Dresselhaus G and Eklund P C 1996 Science of fullerenes and carbon nanotubes (San Diego, CA: Academic Press)

Ferrari A C and Robinson J 2000 J. Phys. Rev. B61 14095

Islam S S and Shah Khurshed Ahmad 2006 IEICE Electronic Express (Japan) 25

Javey Aguo, Wang J Q, Lundstrom M and Dai H 2003 Nature 424654

Jorio A et al 2000 Phys. Rev. Lett. 852617

Jorio A, Pimenta M A, Souza Filho A G, Saito R, Dresselhaus G and Dresselhaus M S 2003 New J. Phys. 5139

Journet C et al 1997 Nature 388756

Kastner J et al 1994 Chem. Phys. Lett. 22153

Kong J et al 2000 Science 287622

Kurti J, Zolyomi V, Gruneis A and Kuzmany H 2002 Phys. Rev. B65 165443

Laurent C, Flahaut E, Peigney A and Rousset A 1998 New J. Chem. 296195
Maultzsch J, Reich S and Thomsen C 2001 Phys. Rev. B64 $121407(\mathrm{R})$

Maultzsch J, Reich S, Schlecht U and Thomsen C 2002 Phys. Rev. B65 233402

Namkung M, Namkung J S, Wincheski B, Seo J and Park C 2004 (unpublished)

Rao A M et al 1997 Science 18757

Saito R, Takeya T, Kimura T, Dresselhaus G and Dresselhaus M S 1998 Phys. Rev. B57 4145

Saito R et al 2003 New J. Phys. 5157.1

Sanjuan M L, Anson A and Martinez M T 2004 Phys. Rev. B70 201404 (R)

Shirolshi T, Sawada T, Hosono A and Nakata N 2003 Technical Digest of IVMC

Tan P, Deng Y and Zhao Q 1998 Phys. Rev. B58 5435

Thomsen C 2000 Phys. Rev. B61 4542

Thomsen C and Reich S 2000 Phys. Rev. Lett. 855214

Thomsen C, Rafailov P M, Jantoljak H and Reich S 2000 Phys. Status Solidi (b) 220561

Trans S, Verschueren A and Dekker C 1998 Nature 49394

Tuinstra F and Koenig J L 1970 J. Chem. Phys. 53126

Wei J, Ci L, Jiang B, Li Y, Zhang X, Zhu X, Xu C and Wu D 2003 J. Mater. Chem. 131340

Wooning K et al 2002 Nanoletts 2703

Zhou C, Keng J and Dai H 2000 Phys. Rev. Lett. 845604 